\title{
Modelling the Localization Scheme Integrated with a MAC Protocol in a Wireless Sensor Network
}

\author{
Suman Pandey \\ Assistant Professor \\ KNIT Sultanpur \\ Sultanpur
}

\begin{abstract}
Node localization is one of the major issues of wireless sensor networks that determines the physical positions of nodes. The location data is useful to many communication protocols such as packet routing and sensing coverage in wireless sensor networks. It has been a daunting task to develop a practical algorithm for node localization in an environment where sensor nodes are with limited power, low cost etc. In this paper a novel idea to conserve energy required in a localization algorithm by integrating it with duty cycled MAC protocol in wireless sensor network has been modelled. To model the idea the RSSI localization method and a sensor SMAC protocol to regulate the control of energy wastages has been considered. Finally, every minute aspect of sensor network localization model including the topology, mobility, and channel and propagation schemes with scheduled transmission access, have been discussed in this paper.
\end{abstract}

\section{INTRODUCTION}

The proliferation in processing capability, storing capacity and communication technologies has expanded the application horizon of sensor systems and the pace of growth is being realized in its wide range of applications covering the whole geographic space from sea to space. The performance of wireless sensor systems varies according to the status and varying values of attributes like power consumption of nodes, rate of node's failures, link failure, heterogeneity of nodes, and ability to withstand harsh environmental conditions. Sensor nodes are defined as small computers having limited resources such as processing unit with limited computational power, limited storage, a communication device like radio transmitter/receiver and finally and most critical energy source [1].

The Wireless HART, IEEE1451, and Zigbee/802.15.4 are some standards for wireless sensor networks developed or being developed by the organizations like WAVE2M, IEEE, Internet Engineering Task force, and International Society of Automation. The main characterizing feature of WSN is its power consumption that defines the lifetime. Algorithms and protocols are required to address issues such as lifetime maximization, robustness and fault tolerance and selfconfiguration.

The fundamental task of sensor network lies in its efficient localization scheme that enhances the capacity of sensor network in terms of efficient routing, energy consumptions in applications like target tracking and location finding etc. With large in number, smaller in size (so minimal in computation and communication abilities), mobility in nodes and with un accessible deployed terrain it is not feasible to provide position configuration manually or attaching a localizing component such as GPS in each node.

A self position finding system is required so that each node localizes itself in the network by using some references (some anchors are used to initialize the localization procedure). The major constraint of wireless sensor network lies in its energy provision system that is limited and this major factor determines the life and hence the usability of the network. This is reason the research community is putting potential effort to develop protocols or algorithms addressing this issue by efficient utilization of energy and thus reducing the energy consumption. With this line of action a model of localization system which employs a duty-cycled media access protocol to reduce the energy wastages by reducing collision and avoiding overhearing, has been proposed. The rest of the paper is organized as follows. The section II covers the work related with the proposals. In the section III, various design parameters of localization method has been discussed. Section IV presents the proposed model of localization scheme. The section $\mathrm{V}$ discusses the performance of the model using probability theory and finally the paper is concluded with future suggestions of the present work

\section{RELATED WORK}

The contribution of research fraternity in the field of localization can be grouped into two categories named as range-based for fine-grained and range-free for coarse-grained wireless sensor network [2].In range-based categories [3-6] the localization algorithm work upon distance or angle measurements between pair of nodes to estimate the position of a sensor node. Some major location algorithms falling in this category require extra hardware to measure the time in Time Difference of Arrival (TDoA), the angle in Angle of Arrival (AoA), and signal strength in Received Signal Strength Indication (RSSI). In the other category of range-free localization these normally make use of parameters e.g. proximity information, less accurate distance estimation to infer the location of a sensor node. Some of the important localization techniques that fall into this are Centroid, Multidimensional Scaling Map (MDS-MAP), Amorphous localization algorithm.

Regarding node localization A. Savvides et al. [3] proposed an Ad-Hoc Localization System. In this anchor nodes broadcast location data over radio frequency $[R F]$ and ultrasound signals concurrently and a node receives the RF signal earlier than the ultrasound signal. By measuring the time difference the node computes its distance from the anchor but in this time difference may not be accurate due to several natural occurring obstructions such as multi-path fading, noise 
interference etc. TDoA location system for resource constrained WSN deteriorates on ground of more energy consumption and expensive hardware.

In localization technique based on angle-of-arival the Ad hoc Positioning Systems (APS) proposed by Niculescu and Nath [4] each node computes its position by angular separation between anchors and itself. Another proposal made in line by Kusy et al. [5] is known to be an enhanced version of Radio Interferometric Positioning System (RIPS). In this method each pair of neighbouring nodes transmits radio signals simultaneously and almost at the same frequencies for creating a low-frequency interference signal. The combined signal produced by interference of the two signals the relative location is evaluated by measuring the relative phase offset. In the literary work it has been shown by the authors that selection of frequencies and multi-path and ground reflection affects the accuracy of the distance measured.

C. Papamanthou et al. [6] worked and designed a positioning system using the received signal strength indicator the RSSI method. First, authors have formulated the probability density function of the actual position of the sensor node within a certain region, given that the power received from the beacons and is modelled with RSSI.

The work of Bulusu et al. [7] has been of a potential advantage in field of range-free methods for localization. In this methodology certain number (fixed) of reference nodes with overlapping communication range are taken and these nodes broadcast periodically their messages containing their own position information and if it is received by neighbors then they store and maintain all received messages. By using this position information, node self computes its position that is expected to be at the centroid of all collected reference nodes. A simple location scheme achieves more accuracy in estimated location in case of higher number of reference nodes with regularly distributed.

Y. Shang et al. [8] have developed a Multidimensional Scaling (MDS-MAP) in range free location algorithms. It is defined with three basic steps. In first step, each node prepares a distance matrix with shortest distance of its neighboring nodes based on connectivity information. Then in the second step, with MDS technique the distance matrix is converted into a relative map. This map defines a location for each node on the plane. Finally, the relative map is transformed through various stages of scaling, rotation, and reflection to give the true location of the nodes. The idea behind the transformation is to minimize the sum of squares of the errors in computation of the position estimation.

In the category of range free location technique Nagpal et al. added a new algorithm named as an Amorphous Localization Algorithm [9]. In this method a beacon always initializes the process of localization by broadcasting message containing its position as (Xbeacon,Ybeacon) and a counter variable set to one. Each node upon receiving the message stores the value of the counter and sends the message further with increased counter value by one. With this each node maintains the minimum counter value received and ignores the messages of larger counter values. This prevents message from being flooded or duplicated in network. After estimating distances (in hops) to at least three beacons, the node computes its location by applying multilateration technique. This algorithm requires sufficient number of beacon nodes to work with.
From above discussion it has been concluded that most of the proposed (or being implemented) localization methods demands for specialized hardware unit such as antenna arrays, global positioning system (GPS) etc. But a few do not require such expensive components are those based on Received Signal Strength Indicator (RSSI). The RSSI based method exploits the inbuilt feature of commonly used nodes (e.g. mica2) to measure radio signal strength received at the node. In spectrum of the factors that views the RSSI based methods produces localization with limited accuracy and energy wastages. This limited accuracy is due to natural occurring phenomenon e.g. multi-path fading, noise interference, the very basic feature of wireless communication is uncontrolled and that is why we have considered a RSSI based localization technique. The energy wastages for localization is reduced through integrating it with a media access protocol for the case it is a sensor MAC.

\section{THE DECISION ATTRIBUTES OF A LOCALIZATION SYSTEM}

From the above discussion it has been observed that generally, a localization method involves two passes. The first pass collects data from surroundings needed for position determination in the application scenario and in second pass the node computes its position by using the collected data in $1^{\text {st }}$ pass. Thus the complexity or accuracy of a localization system can be dependent on following decisions variables such as:

1. The type of ranging data e.g. time, power, angle, link status is used to measure the distance or connectivity with reference nodes (whose position is known).

2. The status of error distribution in above mentioned ranging data such as parameters of RF propagation, the signalling scheme, the modulation scheme and the accuracy of the devices involved in transmission and reception, environment variables (noise, interference from other in cellular cells) etc.

3. The topology or the dimension of deployed WSN and the ratio of anchor nodes (whose position is to be computed) to the normal nodes.

4. If the application scenario of WSN involves mobility of the nodes or not.

5. The nature of computational method whether based on optimization or rely on direct formula.

6. The nature of location algorithm whether it computes position centrally or it follows distributed or hybrid approach.

Thus it is observed that the various factors affect the localization algorithm and in general a single algorithm cannot satisfy the needs of a wide application range of WSN.

\section{SYSTEM MODEL OF PROPOSED LOCALIZATION ALGORITHM}

The underlying methodology of the proposed localization system requires following schemes to work with. The performance of proposed method will be discussed after defining the general assumptions that are made about WSN and then followed by subsequent schemes to be considered. 


\subsection{General Assumptions about Network}

1. The sensor nodes are placed randomly on a network area which is assumed to be two dimensional. The sensor nodes do not know their topological information or their physical position. The nodes may move within the boundary.

2. It is supposed that all sensor nodes are of same design constructs such as initial power, computational capability and communication strength or coverage. The nodes lying within communication range of a node are called its neighbours i.e. the nodes at one-hop distance.

3. It is also a part of assumption that initially some beacons or anchors are placed within deployed area such that at least 3 of them are neighbours of each other. Some additional references may be anywhere within the network. The design constructs of beacons or anchors are similar with other nodes except they know their positions.

4. The paper defines that network is connected and any active node can communicate with the sink or base node either in single hop or in multiple hops.

5. If Euclidean distance of two is not larger than the communication range it can directly communicate and exchange their data.

\subsection{Basic Scheme of S-MAC (Sensor Medium Access Control) Protocol}

The basic scheme of S-MAC [10] is to off the receiver if a node is not engaged in any transmission and reception for a period. This saves energy that is wasted in overhearing and idle listening after hearing a RTS or CTS frame for a time (that is the period of data transmission or reception of at least one sensor node other than itself).To implement this idea the S-MAC scheme periodically goes into sleep state. This is a listen-sleep cycle and models a time frame. The ratio of listen period to sleep period defines duty cycle of radio. This, dutycycle may be varied in accordance with the application scenarios. For simplification it is kept constant for all nodes in WSN. Period of cycle defines a time frame consisting of time slots. According to work specified, the listen time is divided into synchronization period (some fixed initial time slots) and data transmission/reception period (following slots). To be successful it requires periodic synchronization among competing nodes to cancel the system clock differences and also to reduce contention. To reduce the synchronization error all time-stampings are relative instead of absolute. To avoid contention, the neighboring nodes should listen at the same time and also sleep at the same time. To implement this thought the S-MAC lets the neighboring nodes follow a common schedule of listen and sleep. This is managed and maintained by neighbour discovery process that prepares a schedule. The nodes informs of their schedule to neighbours by broadcasting its schedule. If more than one node wants to talk to a node (destination), they all contend for medium using contention mechanism of IEEE 802.11 using RTS (request to send) and CTS(clear to send) packets. Thus each node stores and maintains a schedule table containing its neighbours' schedule. Firstly, a node listens for certain time slots (carrier sense duration cs1) and if do not hear a schedule of others it broadcasts its schedule in a SYNC message, informing the neighbours that after $t$ seconds it will enter into sleep state.
After completing the synchronization if it has data packets in its queue it re-enters into carrier sense (cs2 stage) and finding the channel free for that duration, it sends RTS packet and after receiving CTS packet from the receiver sensor (in listening period) it sends the data packets and after receiving ACK (acknowledgement) packets it goes into sleep state and in next cycle it repeats the process for rest of the packets. In a case if RTS/CTS gets collided or lost it goes into sleep state and tries in next cycle.

\subsection{Basic schemes of RSSI and Trilateration Method}

In this method basically reference nodes (whose position coordinates are known) broadcast its position data with its identification(Idi) as $\{(x i, y i), I d i\}$.The neighbouring nodes upon receiving this message store it and compute its distance (dji) from that anchor node (Idi) by using RSSI value of received broadcast signal. Whenever a node receives such position data from at least three nodes this node computes its position coordinate by using trilateration method. Figure.1 depicts a wireless sensor network in which beacon nodes sends their position data to the neighbouring nodes and upon receiving data at least from three beacons a sensor node estimates its position and become a reference node that further broadcasts its position. In this way the unknown nodes finally gets localized.

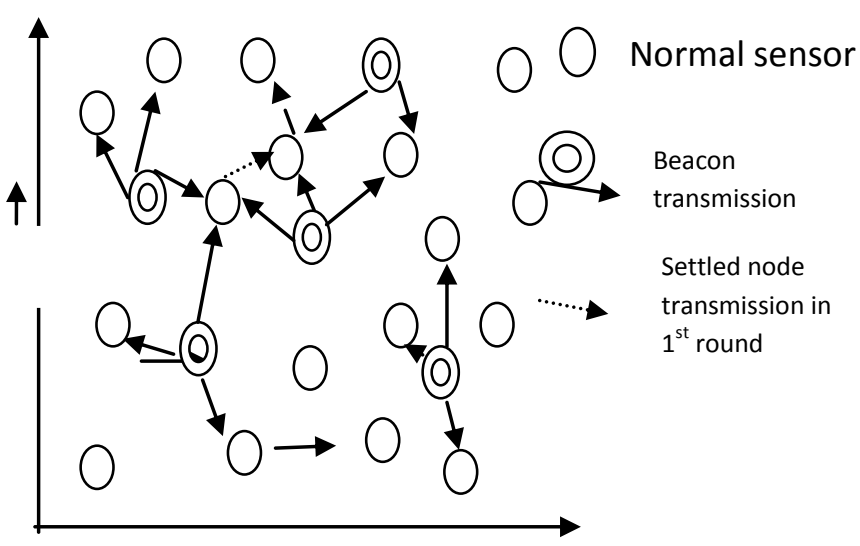

Figure: 1 Wireless sensor network

\subsection{Work flow of Proposed of localization method}

The Figure. 2 describes the basic steps to be carried out in localization process. These steps can be grouped into collection phase of location data and then applying a triangulation method to compute the position of sensor node. These are passes where synchronization with duty controlled media access protocol could perform better in terms of reduced latency time. During various computations i.e. transmission as well as position calculation, the channel by going into sleeping mode (controlled) can reduce the energy wastages in collisions and overhearing. 


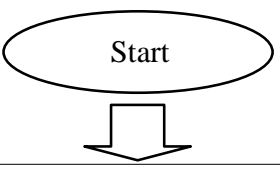

Initialization process:

1.Assigning identifiers to each sensor

2.Group the sensor nodes into two sets

$\mathrm{S}$ (whose position is to be estimated) and

$\mathrm{U}$ (with known position).

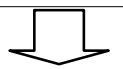

Reference nodes broadcast their position data and the nodes lying in its vicinity receive it and store it with Its RSSI value. Thus nodes, with RSSI value compute its distance from that reference nodes using fris equation.

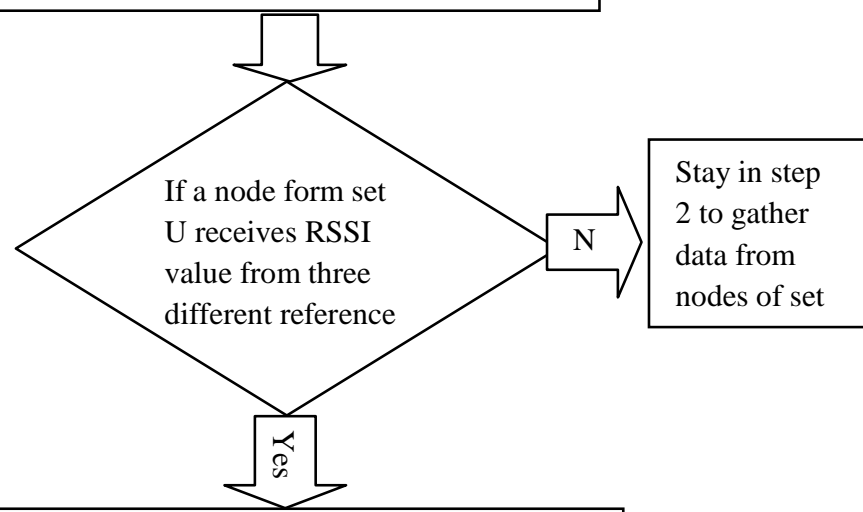

Position Computaion: Using trilateration or multilateration.

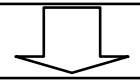

Updation Process: the node localized recently is deleted from set $\mathrm{U}$ and added to set $\mathrm{S}$ and now it will act as reference node.

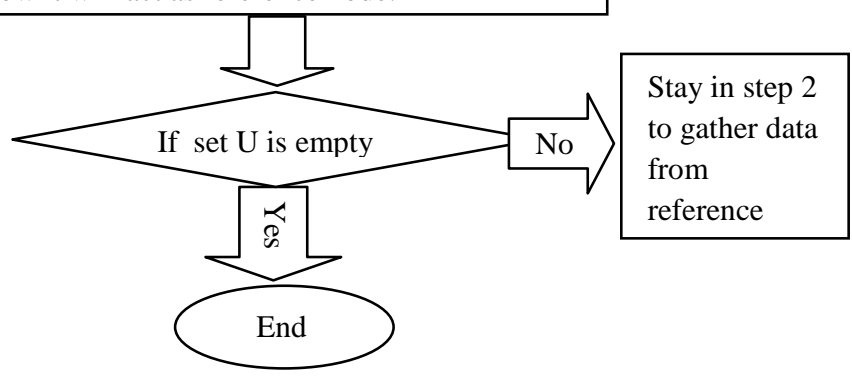

\section{THE ANALYTICAL MODEL OF S- MAC SCHEME REGULATING THE LOCALIZATION METHOD}

On the basis of analytical model [11-12] in this section of paper we analyze the performance of mac scheme. For this it uses discrete time Markov chain model to study the status of a sensor node in a WSN application. In figure. 2 it has been shown the change of its state with time and to represent this scenario a stochastic process $S(t)$ is taken into consideration.

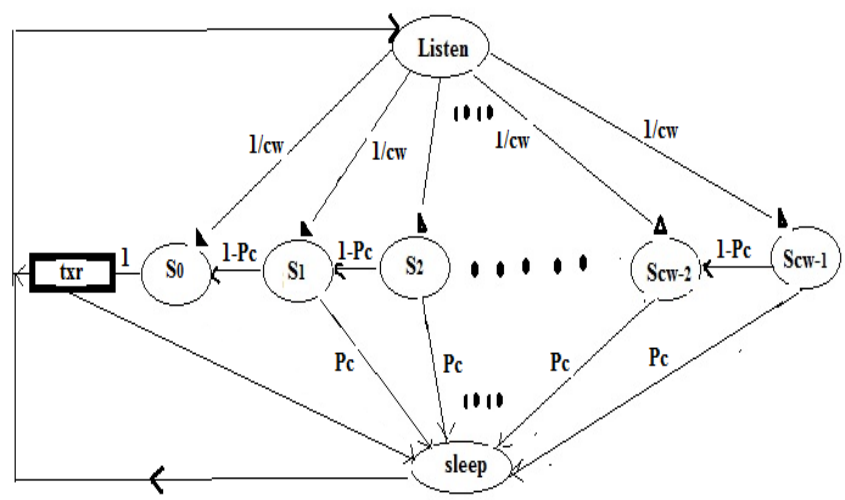

Figure: 2 Markov Chain for Sensor Node's States under SMAC Scheme

Consider a single-hop wireless sensor network with a sink (acting as cluster head). To have communication with reduced contention and overhearing among the competing sensor nodes the paper considers the S-MAC scheme. In underlying scheme each node rests periodically in either Listen state or Sleep state. The listen period starts with SYNC followed with a DATA period and then goes into sleep state. For the analysis assume that sync period is over and entering into data transmission/reception period. To have successful transmission it first goes into carrier sensing phase. This is implemented by picking a random period from contention window $(0, \mathrm{CW})$ and sense the carrier for that period. The $\mathrm{CW}$ defines a range of slots with minimum number of time-slots to a maximum number of time-slots. For simplicity assume that each node has a fixed range of $\mathrm{CW}$. So before going into data transmission, it backs off itself for that random period. Each node has a fixed probability equals to $1 / \mathrm{CW}$ for going into any of the stage of backoff counter (bc) and starting from that stage it senses the carrier for a slot if it is free it then decrease it bc value by one and enters into new stage and repeats itself until it reaches into a stage with backoff value 0 and it shown by stage $\mathrm{S}_{0}$. In any stage if it senses other's transmission it goes into sleep state and tries in next cycle with new value. With the change of bc value we have shown the status of node $\mathrm{S}(\mathrm{t})$ with time. Thus, $\mathrm{S}(\mathrm{t})$ can be modelled with discrete-time Markov chain as shown in the figure. The possible transition probabilities for only one step are shown with following recurrence formulae.

Let Pc is the probability of finding the channel busy. So (1Pc) is the probability of finding the channel free and going into new stage with value of bc decreased by one.

$$
\left\{\begin{array}{lc}
\mathrm{P}(\mathrm{k}-1 / \mathrm{k})=\mathrm{pc} / \mathrm{cw}+1-\mathrm{pc} ; & \mathbf{k} \varepsilon[1, \mathrm{cw}-1] \\
\mathrm{P}(\mathrm{i} / \mathrm{k})=\mathrm{pc} / \mathrm{cw} ; & \mathbf{k} \varepsilon[1, \mathrm{cw}-1], \mathbf{i} \varepsilon[0, \mathrm{cw}-1], \mathrm{i} \neq \mathrm{k}-1 \\
\mathrm{P}(0 / \mathrm{k})=1 / \mathrm{cw} ; & \mathrm{k} \varepsilon[0, \mathrm{cw}-1]
\end{array}\right\}--(1)
$$

With above known set of transition probabilities we are interested in finding out transmission probability of a sensor node.

Except the last stage or bc with maximum value we can say that each node status $S_{k-1}$ is obtained by $S_{k}$ with probability 1pc and with a probability $\mathbf{x}$ from any other possible status of node that is from $\mathrm{S} 0$ to $\mathrm{Scw}-1$. So expression for $\mathrm{x}$ is

$\mathrm{x}=\left(\mathrm{S}_{0} / \mathrm{cw}\right)+\left[\mathrm{pc} / \mathrm{cw}\left(\mathrm{S}_{1}+\mathrm{S}_{2}+\mathrm{S}_{3} \ldots \ldots \ldots . . . \mathrm{S}_{\mathrm{cw}-1}\right)\right] \ldots$ 
So from any stage (except the stage Scw-1) it can be reached to state $S_{0}$. So probability to reach in state $S_{0}$ or the transmission probability $\mathrm{P}_{\mathrm{t}}[13]$ can be expressed as follows:

$$
\left\{\begin{array}{l}
\mathrm{P}_{t}=\mathrm{s} 0 ; \\
\mathrm{S}_{0}=(1-\mathrm{pc}) \mathrm{S}_{1}+\mathrm{X} \\
\mathrm{S}_{1}=(1-\mathrm{pc}) \mathrm{S}_{2}+\mathrm{X} \\
\mathrm{S}_{2}=(1-\mathrm{pc}) \mathrm{S}_{3}+\mathrm{X} \\
\cdot \\
\cdot \\
\mathrm{Scw}_{-2}=(1-\mathrm{pc}) \mathrm{Scw}-1+\mathrm{X} \\
\mathrm{Scw}-1=\mathrm{X}
\end{array}\right.
$$

$\mathrm{S}_{0}=(1-\mathrm{pc}) \mathrm{S}_{1}+\left[\left(\mathrm{S}_{0} / \mathrm{cw}\right)+\left[\mathrm{pc} / \mathrm{cw}\left(\mathrm{S}_{1}+\mathrm{S}_{2}+\mathrm{S}_{3} \ldots \ldots \ldots . . . \mathrm{S}_{\mathrm{cw}-1}\right)\right]\right]$

From the probability theorem $\mathrm{X}=\left(\mathrm{S}_{0} / \mathrm{cw}\right)+\left[\mathrm{pc} / \mathrm{cw}\left(\mathrm{S}_{1}+\mathrm{S}_{2}+\right.\right.$ $\left.\left.+\mathrm{S}_{3} \ldots \ldots \ldots . . . . \mathrm{S}_{\mathrm{cw}-1}\right)\right]$

$\mathrm{X}=\left(\mathrm{S}_{0} / \mathrm{cw}\right)+\left\{\mathrm{pc} / \mathrm{cw}\left(1-\left(\mathrm{S}_{0}\right)\right\}\right.$

$\left\{\mathrm{S}_{0}(1-\mathrm{pc})+\mathrm{pc}\right\} / \mathrm{cw}$

$\mathrm{S}_{0}=(1-\mathrm{pc})\left((1-\mathrm{pc}) \mathrm{S}_{2}+\mathrm{X}\right)+\mathrm{X}$

$=(1-p c)^{\wedge} 2 S_{2}+(1-p c) X+X$

$=(1-p c)^{\wedge} 3 S_{3}+(1-p c)^{\wedge} 2 X+(1-p c) X+X$

$=(1-\mathrm{pc})^{\wedge}(\mathrm{cw}-1 \quad) \quad \mathrm{Scw}-1+(1-\mathrm{pc})^{\wedge}(\mathrm{cw}-2 \quad) \mathrm{X}+\ldots \ldots .+(1-$ pc $)^{\wedge} 2 S_{2}+(1-p c) X+X$

$=(1-p c)^{\wedge}(\quad c w-1 \quad) X+(1-p c)^{\wedge}(\quad c w-2 \quad) \quad X+\ldots \ldots \ldots \ldots . .+(1-$

pc $)^{\wedge} 2 \mathrm{~S}_{2}+(1-\mathrm{pc}) \mathrm{X}+\mathrm{X}$

$=\mathrm{X}\left((1-\mathrm{pc})^{\wedge}(\mathrm{cw}-1)+(1-\mathrm{pc})^{\wedge}(\mathrm{cw}-2)+(1-\mathrm{pc})^{\wedge}(\mathrm{cw}-3)+\ldots \ldots \ldots .\right.$.

$+(1-\mathrm{pc})+(1-\mathrm{pc})$

$\left.=\mathrm{X}\left[1-(1-\mathrm{pc})^{\mathrm{ccw})}\right] /(\mathrm{pc})\right]$

$\left.\mathrm{S}_{0}=\left\{\mathrm{S}_{0}(1-\mathrm{pc})+\mathrm{pc}\right\} / \mathrm{cw} *\left[1-(1-\mathrm{pc})^{(\mathrm{cw})}\right] /(\mathrm{pc})\right]$

\begin{tabular}{c}
$\mathbf{P}_{\mathbf{t}}=\frac{\mathrm{pc}\left[1-(1-\mathrm{pc})^{\mathrm{cW}}\right]}{\left[\mathrm{cwpc}-(1-\mathrm{pc})\left[1-(1-\mathrm{pc})^{\mathrm{w}}\right]\right]}$ \\
\hline
\end{tabular}

$\mathbf{P}_{\mathbf{t}}$ is the transmission probability it can be said that value of SMAC parameters such as backoff mechanism, contention window size, the probability of competing transmissions, queuing behaviour, arrival pattern of traffic affect the data collection phase. Tuning with required database it can be adjusted to give better performance. With this we see that basis steps of data gathering and computation of position can be controlled by underlying media access control protocol to get improved performance. The parameters from physical layer such as signal strength measurement technique, path loss model will also affect the localization procedure. Thus we conclude that efficiency of localization system can be further improved with optimized media access protocol.

\section{CONCLUSION AND FUTURE SCOPE}

Enhancing the life of sensor networks by reducing the energy consumption is an important issue. In this paper, we have proposed a localization model which by use of duty-cycled media access control protocol gives better performance in terms of reduction in the latency time in estimating the position of sensor nodes. This time is basically dependent upon how faster it gathers the required information with little redundancy and then by using triangulation or multilateration method it computes the position. With this model we have proposed an energy efficient localization protocol that reduces the wastage of energy. The model on probabilistic approach concludes for enhancement in the network's life. In future this is going to help design QoS in achieving the network localized. An optimized media access protocol specifically for localization process can be used to achieve quality of service in target tracking etc. In future, work can be focussed on other factors such as maximizing the localization coverage, reduction in the time for getting localized and reduction in the communication cost of localization system.

\section{REFERENCES}

[1] I. F. Akyildiz, W. Su ,Y. Sankarasubramaniam, E. Cayirci. "Wireless Sensor Networks: a survey, Computer Networks 38 (2002) 393-422.

[2] S. Halder, A. Ghosal and S. DasBit, "A Pre-determined Node Deployment Strategy to Prolong Network Lifetime in Wireless Sensor Network," Computer Communications, 34(11), pp. 1294-1306, 2011.

[3] M. Rudafshani and S. Datta, "Localization in Wireless Sensor Networks,” Proc. of IPSN, pp. 51-60, 2007.

[4] A. Savvides, C. C. Han and M. B. Strivastava, "Dynamic Fine Grained Localization in Ad-Hoc Sensor Networks,' Proc. of MOBICOM, pp. 166-179, 2001.

[5] D. Niculescu and B. Nath, "Ad Hoc Positioning System Using AOA," Proc. of IEEE INFOCOM, pp. 1734-1743, 2003.

[6] B. Kusy, A. Ledeczi, M. Maroti and L. Meertens, "Nodedensity Independent Localization," Proc. of IPSN, pp. 441-448, 2006.

[7] C. Papamanthou, F. P. Preparata and R. Tamassia, "Algorithms for Location Estimation Based on RSSI Sampling," Proc. of ALGOSENSORS, LNCS 5389, pp. 72-86, 2008.

[8] N. Bulusu, J. Heidemann and D. Estrin, "GPS-less Low Cost Outdoor Localization for Very Small Devices," IEEE Personal Communications Magazine, 7(5), pp. 28 34,2000 .

[9] Y. Shang, W. Ruml, Y. Zhang and M. P. J. Fromherz, "Localization from Mere Connectivity," Proc. of MOBIHOC, pp. 201-212, 2003.

[10] R. Nagpal, H. Shrobe and J. Bachrach, "Organizing a Global Coordinate System from Local Information on an Ad Hoc Sensor Network", Proc. of IPSN, LNCS 2634, pp. 333-348, 2003.

[11] Wei Ye, John Heidemann, Deborah Estrin, “ An EnergyEfficient MAC Protocol for Wireless Sensor Networks" IEEE Infocom 2002, pages 1567, June 2002.

[12] Ou Yang, Wendi Heinzelman "Modeling and Performance analysis for S-MAC with Retransmissions in Multihop Wireless Sensor Networks"

[13] J. He, Z. Tang, H. Chen, and Q. Zhang, "An accurate and scalable analytical model for IEEE 802.15.4 slotted SMA/CA networks, in IEEE Trans. Wireless Communications, vol. 8, no. 1, Jan. 2009, pp. 440-448.

[14] K. Davey. "Sensitivity and optimization for shape and non-linear boundary conditions in thermal boundary elements", International Journal for Numerical Methods in Engineering, 2003-,pp.553-587 